\title{
Observation of robust energy transfer in the photosynthetic protein allophycocyanin using single-molecule pump-probe spectroscopy
}

\section{Raymundo Moya}

Massachusetts Institute of Technology

\section{Audrey Norris}

Massachusetts Institute of Technology

\section{Toru Kondo}

Tokyo Institute of Technology

Gabriela Schlau-Cohen ( $\sim$ gssc@mit.edu )

Massachusetts Institute of Technology https://orcid.org/0000-0001-7746-2981

\section{Article}

Keywords: photoenergy transfers, photosynthetic organisms, light harvesting

Posted Date: March 12th, 2021

DOI: https://doi.org/10.21203/rs.3.rs-272109/v1

License: (9) This work is licensed under a Creative Commons Attribution 4.0 International License. Read Full License

Version of Record: A version of this preprint was published at Nature Chemistry on January 6th, 2022. See the published version at https://doi.org/10.1038/s41557-021-00841-9. 
1

2

3

4

\title{
Observation of robust energy transfer in the
} photosynthetic protein allophycocyanin using single-molecule pump-probe spectroscopy

\author{
${ }^{1}$ Department of Chemistry, Massachusetts Institute of Technology, \\ 77 Massachusetts Avenue, Cambridge, Massachusetts 02139, USA \\ ${ }^{2}$ Department of Life Science and Technology, Tokyo Institute of Technology \\ *To whom correspondence should be addressed; E-mail: gssc@mit.edu
}

\section{Abstract}

6 Photosynthetic organisms convert sunlight to electricity with near unity quantum efficiency. Absorbed

7 photoenergy transfers through a network of chromophores positioned within protein scaffolds, which

8 fluctuate due to thermal motion. The resultant variation in energy transfer has not yet been measured,

9 and so how the efficiency is robust to this variation, if any, has not been determined. Here, we describe

10 single-molecule pump-probe spectroscopy with facile spectral tuning and its application to the ultrafast

11 dynamics of single allophycocyanin, a light-harvesting protein from cyanobacteria. Using the spectral

12 dependence of the dynamics, energy transfer and energetic relaxation from nuclear motion were dis-

13 entangled. For energy transfer, an asymmetric distribution of timescales was observed. For energetic

14 relaxation, the timescales were slower and more heterogeneous due to the impact of the protein en-

15 vironment. Collectively, these results suggest that energy transfer is robust to protein fluctuations, a

16 prerequisite for efficient light harvesting. 


\section{Introduction}

18 Photosynthetic light-harvesting systems power most life on earth by capturing and directing absorbed

19 energy through networks of protein-scaffolded chromophores [1]. Rapid transfer of the absorbed energy

20 is driven by coupling between the transition dipole moments of the chromophores. Fluctuations of the

21 protein scaffold induce changes in the distances and orientations of the transition dipole moments that

22 can, in turn, change the timescales of energy transfer [2-7]. Despite these fluctuations, energy travels

23 through the light-harvesting systems to reach the reaction center with near-unity quantum efficiency [1,

24 8]. Heterogeneity in the timescales of energy transfer, i.e, the impact of protein fluctuations on light

25 harvesting, has not yet been characterized.

26 Over the past decades, single-molecule methods have been a powerful approach to characterize hetero-

27 geneity in biological, chemical, and material systems, including photosynthetic light-harvesting proteins

28 [9-12].More recently, single-molecule pump-probe spectroscopy (SM2P) emerged as a technique to

29 resolve femtosecond processes, such as energy transfer. SM2P maps femtosecond dynamics onto flu-

30 orescence intensity using two ultrafast isoenergetic pulses to generate a pump-probe like excitation,

31 where the temporal resolution is from the delay time between pulses (Fig. 1a) [13-16]. This tech-

32 nique has been applied to various ultrafast processes in single molecules, including coherent wavepacket

33 oscillations and relaxation within the excitonic states of light-harvesting proteins from purple bacteria

34 [16-18]. The complexity of the ultrafast dynamics of light-harvesting proteins has, however, obfuscated

35 the heterogeneity specific to energy transfer. Furthermore, most of these measurements lacked spectral

36 dependence, which provides an additional axis to help disentangle the contributions associated with each

37 process.

38 The cyanobacterial light-harvesting protein, allophycocyanin (APC), contains strongly coupled dimers

39 of chromophores that serve as a minimal system to examine photosynthetic energy transfer (Fig. 1b).

40 Ensemble ultrafast measurements found complex kinetics of energy transfer within APC, potentially due

41 to heterogeneous timescales [19-28]. Consistently, single-molecule fluorescence measurements identified

42 heterogeneous photophysical states that arose from fluctuations of the protein scaffold [29-34], yet

43 their impact on energy transfer could not be resolved due to the limited time resolution of fluorescence

44 measurements. 
45 Here, we report SM2P with facile spectral tuning across the visible region and perform SM2P measure-

46 ments on APC, the homologous protein C-phycocyanin (CPC), and a chromophore in solution. Based

47 on the spectral dependence of the dynamics and the concomitantly-measured fluorescence lifetimes, the

48 distributions of energy relaxation and energy transfer timescales were separated for APC. The energy

49 transfer timescales were centered at $\sim 150 \mathrm{fs}$. While the mean of the distribution agreed with the longer

50 timescale from ensemble measurements, the median of the distribution was $\sim 100 \mathrm{fs}$ shorter, suggesting

51 that ensemble values may be lengthened due to slow sub-populations. The asymmetric nature of the

52 distribution gives rise to non-mono-exponential behavior in the ensemble, such as the multi-exponential

53 energy transfer observed in previous measurements $[22,28]$. Broad distributions of energetic relaxation

54 timescales were observed for both APC and CPC, which comparison to choromophores in solution in-

55 dicated arises from the protein structure. Furthermore, slow energetic relaxation was observed in APC,

56 enabling energy transfer to precede, and thus likely be unaffected by, the heterogeneity in the relaxation

57 timescales. Together, these results demonstrate that rapid energy transfer is maintained despite fluc-

58 tuations of the protein, which may play a role in the high quantum efficiency of photosynthetic light

59 harvesting.

\section{Results}

\section{Ultrafast dynamics in APC}

62 We used SM2P to determine the distribution of ultrafast energetic relaxation timescales in APC. APC

63 forms a trimer where each monomer contains two protein subunits, known as $\alpha$ and $\beta$, that each bind a

64 phycocyanobilin chromophore, which are positioned on distal ends of the monomer ( $\sim 5 \mathrm{~nm}$ apart) [35,

65 36]. The structural model of trimeric APC is shown in Fig. 1b. Upon trimerization, complementary

66 chromophores on different monomers are brought close together ( $\sim 2 \mathrm{~nm}$ apart) to generate the dimer

67 shown in Fig. 1b, inset $[35,36]$. The trimers assemble into the central core of the primary cyanobacterial

68 light-harvesting structure, known as the phycobilisome [36] The linear absorption spectrum of APC is

69 shown in Fig. 1c with the calculated absorption profiles for the underlying states [22]. The higher energy

70 state has a broad absorption and the lower energy state has narrow absorption with a clear vibrational

71 mode at $\sim 1,600 \mathrm{~cm}^{-1}$ above the primary transition. Although the electronic coupling between the $\alpha$ 
a
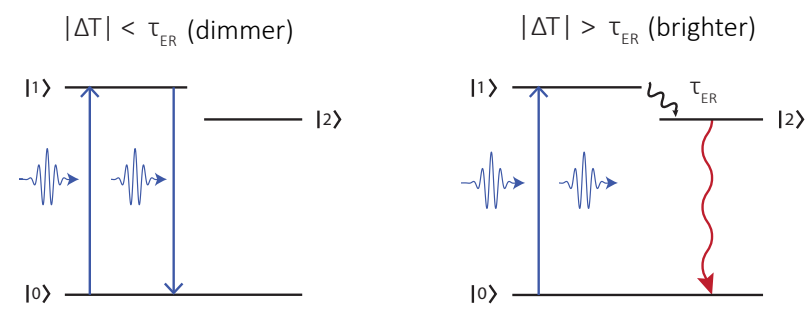

b

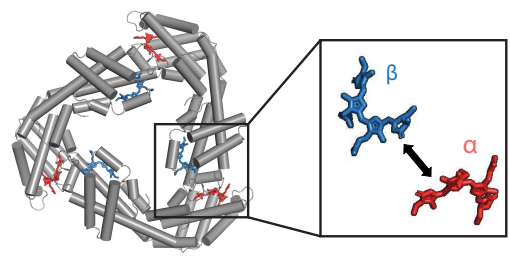

C

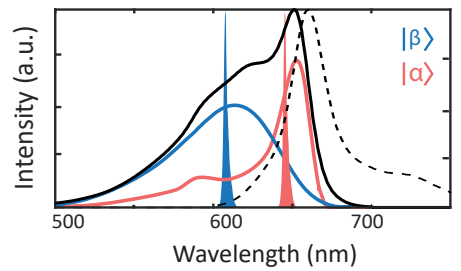

d

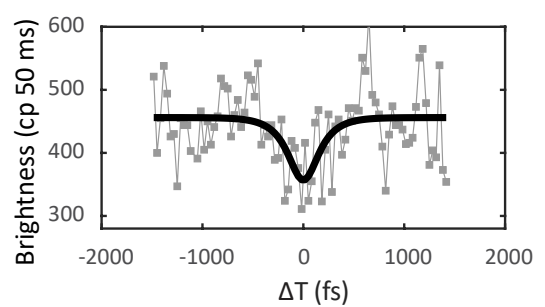

e

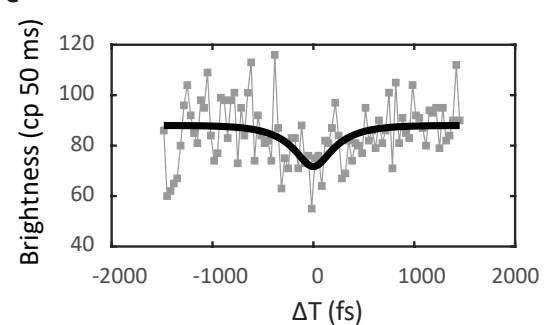

$\mathbf{f}$

g
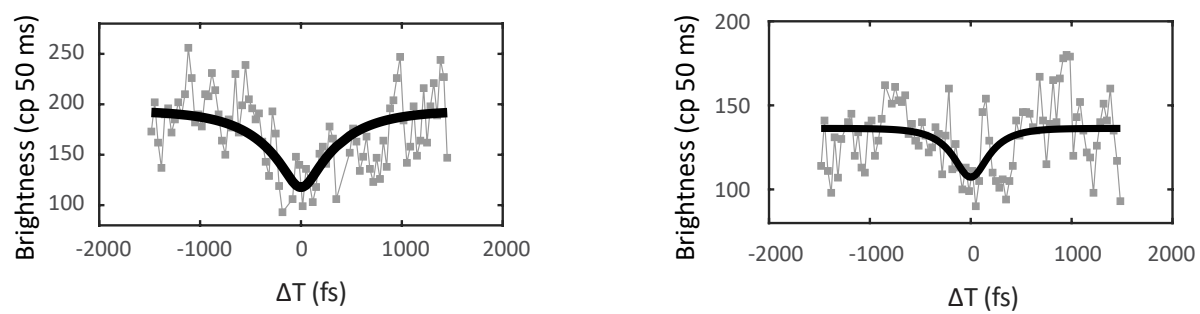

Figure 1: Single-molecule pump probe (SM2P) experiments on allophycocyanin: (a) Two laser pulses are focused onto the sample where energy relaxes between two excited states (a donor, $|1\rangle$, and an acceptor, $|2\rangle$ ) with a timescale, $\tau_{E R}$. If $\Delta \mathrm{T}$ is less than $\tau_{E R}$ (left), pulse 2 stimulates emission, decreasing the fluorescence intensity. If $\Delta \mathrm{T}$ is greater than $\tau_{E R}$ (right), fluorescence emission occurs, increasing the fluorescence intensity. Scanning the time delay from negative to positive delays produces a dip like shape where the width of the modulation is governed by $\tau_{E R}$. (b) Structural model of allophycocyanin with the $\beta$ (blue) and $\alpha$ (red) chromophores (PDBID 1ALL). (c) Absorption (solid) and emission (dashed) spectra are shown with the $610 \mathrm{~nm}$ and $645 \mathrm{~nm}$ excitation in blue and red, respectively. The individual spectra for the $\alpha$ and $\beta$ chromophores are shown in blue and red [22]. Representative SM2P traces (gray) of allophycocyanin with fits (black) for $610 \mathrm{~nm}$ excitation with decay time constants of $148 \pm 15$ and $225 \pm 70 \mathrm{fs}$ are shown in (d) and (e), respectively and for $645 \mathrm{~nm}$ excitation with decay time constants of $371 \pm 46$ and $185 \pm 39 \mathrm{fs}$ are shown in (f) and (g), respectively.

72 and $\beta$ chromophores leads to excited states that are a linear combination of the excited states of the

73 individual chromophores, the large energy gap means that the higher energy state is dominated by the

$74 \beta$ chromophore and the lower energy one by the $\alpha$ chromophore $[19,22]$. Thus, here we refer to the 75 states based on their dominant contribution. 
76 The SM2P apparatus was constructed with a tunable excitation laser and a single-axis prism compres-

77 sor to enable straightforward wavelength changes, which was used to investigate the dynamics of the

78 individual chromophores. SM2P experiments on APC were performed with an excitation laser centered

79 at $610 \mathrm{~nm}$ and $645 \mathrm{~nm}$, which were selected to predominantly excite the $\beta$ and $\alpha$ chromophores re-

80 spectively (Fig. 1c). In SM2P, the first saturating pulse drives damped Rabi oscillations between the

81 ground state $(|0\rangle)$ and the initially excited state $(|1\rangle)$, resulting in an equal probability of population in

82 both states after interaction with the pulse. Population in $|1\rangle$ relaxes to the off-resonance state $(|2\rangle)$

83 with a timescale determined by the microscopic properties of the sample. The second saturating pulse

84 drives the same Rabi oscillations between $|0\rangle$ and $|1\rangle$, but does not interfere with population out of

85 resonance (i.e., in $|2\rangle$ ). On timescales longer than the relaxation time (Fig. 1a, right), the second Rabi

86 oscillation provides another opportunity to populate $|1\rangle$ and subsequently transfer to $|2\rangle$, increasing the

87 population and thus fluorescence from $|2\rangle$. By detecting fluorescence from $|2\rangle$ and scanning the delay

88 from negative to positive times, an SM2P trace is recorded that shows a dip-like shape, which can be

89 fit to extract the timescale of energy relaxation between states.

90 Representative SM2P traces are shown for primarily $\beta$ excitation at $610 \mathrm{~nm}$ (Fig. 1d,e) and primarily $91 \alpha$ excitation at $645 \mathrm{~nm}$ (Fig. 1f,g). While these $\beta$-excitation traces exhibit timescales that are not 92 statistically different ( $148 \pm 15$ and $225 \pm 70 \mathrm{fs}$ in Fig. $1 \mathrm{~d}$ and e, respectively), the $\alpha$-excitation traces differ 93 significantly ( $371 \pm 46$ and $185 \pm 39$ fs in Fig. $1 \mathrm{f}$ and g, respectively), providing an initial demonstration 94 of the ability of SM2P to uncover heterogeneity in ultrafast dynamics.

\section{Energy transfer in APC}

96 In APC, rapid energy transfer occurs within the chromophore dimers [26]. Energy transfers from the 97 higher energy $\beta$ chromophore to the lower energy $\alpha$ chromophore, and so can be studied using the $\beta$ 98 excitation data. While slower (picosecond) energy transfer also occurs between dimers, it is outside the 99 timescale measured here. Along with energy transfer, both chromophores undergo energetic relaxation 100 on a similar timescale due to nuclear motion. SM2P measures the overall energy relaxation timescale,

101 which includes both energy transfer and energetic relaxation. Under the high excitation fluences of $102 \mathrm{SM} 2 \mathrm{P}$, the chromophores photodegrade into quenching radical cations that decrease the fluorescence

103 lifetime. Photodegradation generally begins on the lower energy $\alpha$ chromophore, likely because it 
a

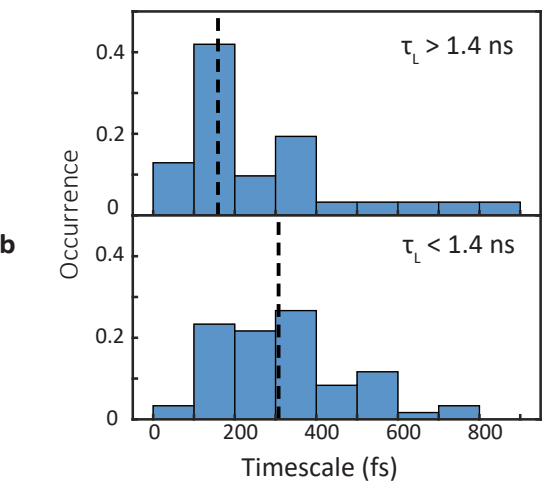

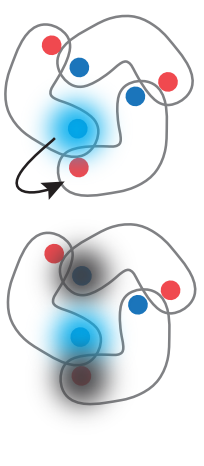

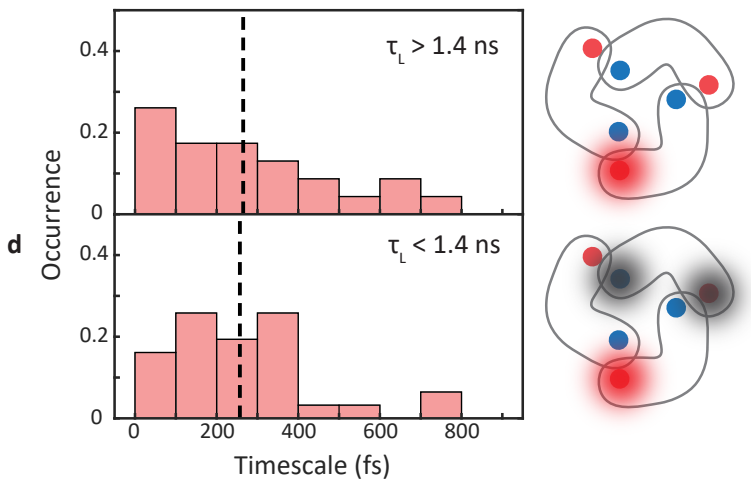

Figure 2: Distributions of energetic relaxation and energy transfer timescales for APC. Histograms of the energy relaxation timescales were constructed from the $\beta$ excitation data $\left(\lambda_{\text {exc }}=610 \mathrm{~nm}\right.$, blue) for the (a) bright $\left(\tau_{L}>1.4 \mathrm{~ns}\right)$ population and (b) quenched $\left(\tau_{L}>1.4 \mathrm{~ns}\right)$ population and from the $\alpha$ excitation data $\left(\lambda_{\text {exc }}=645 \mathrm{~nm}\right.$, red) for the (c) bright population and (d) quenched population. The median values of the distributions are indicated by the dashed lines. As shown in (a), excitation of the $\beta$ chromophore (blue) leads to energy transfer to the $\alpha$ chromophore (red). As shown in $(\mathbf{b}, \mathbf{d})$, photobleaching of individual leads to a loss of photoactivity and/or conversion into quenchers (dark gray).

104 remains excited for longer than the $\beta$ chromophore due to the relatively long time between energy

105 transfer and fluorescence emission [29,30]. The negligible oscillator strength and/or spectral shifts

106 of the photodegraded chromophore eliminate the rapid $\beta$ to $\alpha$ energy transfer, and so only energetic

107 relaxation due to nuclear motion remains [29, 30, 33, 34].To separate and characterize energy transfer

108 and energetic relaxation, four histograms were constructed from the measured timescales, divided by

109 the excitation wavelength and the concomitantly measured fluorescence lifetime. The histograms are

110 shown in Fig. 2 for $\beta$ excitation $(\mathrm{a}, \mathrm{b})$ and $\alpha$ excitation $(\mathrm{c}, \mathrm{d})$ for bright $\left(\tau_{L}>1.4 \mathrm{~ns}\right)$ and quenched

$111\left(\tau_{L}<1.4 \mathrm{~ns}\right)$ populations, respectively. Statistical parameters for the histograms are given in Table S3

112 in SI Section 5. The 1.4 ns cut off value was selected to separate the photodegraded APC based the

113 fluorescence lifetime distributions (Fig. S6, SI Section 4), consistent with previous work [29, 30, 34].

114 In the $\beta$ excitation data, the histograms have medians of $168 \mathrm{fs}$ for bright APC and $308 \mathrm{fs}$ for quenched

115 APC (Fig. 2a, 2b). Comparison of the two distributions with a permutation test yielded a p-value of

1160.0001 (SI Section 5.4), which establishes with a high probability (99.99\%) that the two distributions

117 are different. In contrast, in the $\alpha$ excitation data, the histograms have medians of $276 \mathrm{fs}$ for bright APC

118 and $257 \mathrm{fs}$ for quenched APC (Fig. 2c, 2d), and comparison of the two distributions showed that they are

119 the same ( $p>0.05$, SI Section 5.4). Similarly, the quenched populations for $\beta$ excitation and $\alpha$ excitation

120 were the same $(\mathrm{p}>0.05$, SI Section 5.4). In agreement with these results, only energetic relaxation is

121 thought to be present for all three of these populations. The statistically significant shorter median 
122 timescale for the bright population of the $\beta$ excitation data, the only histogram in which energy transfer 123 is expected, is consistent with this picture. Collectively, these results establish that the bright population 124 of the $\beta$ excitation data primarily describes energy transfer whereas the other three populations primarily 125 describe energetic relaxation, as illustrated in the right panels of Fig. 2.

126 The distribution of primarily energy transfer timescales shown in Fig. 2a has a median of 168 fs and 127 a mean of $258 \mathrm{fs}$, where the difference between these two values is due to its asymmetric profile. In 128 previous ensemble measurements, energy transfer between the two chromophores was found to occur on $129220 \mathrm{fs}$ and $280 \mathrm{fs}$ timescales, which were speculated to correspond to different conformational states of 130 the protein backbone $[23,24]$. The mean is consistent with these values, and the $\sim 100 \mathrm{fs}$ increase over 131 the median suggests that ensemble measurements are significantly lengthened by small sub-populations 132 with slow transfer. This observation of an asymmetric distribution suggests that traditional theories of 133 photosynthetic energy transfer, which were primarily developed based on mean values [2, 7, 37], may

134 be distorted by these slow sub-populations.

135 An asymmetric distribution of energy transfer rates can not only lengthen the average value, but also 136 influence the functional form of measurements. Whereas a Gaussian distribution gives rise to an energy 137 transfer process well-described by a single-exponential function, an asymmetric distribution can lead to 138 more complex behavior, such as a stretched exponential or the bi-exponential observed previously for 139 APC (SI Section 5.6, SI Fig. S13) [22]. Thus, instead of characterizing distinct processes, ensemble 140 measurements of multi-exponential dynamics may arise from non-Gaussian microscopic heterogeneity, 141 similar to the distribution of energy transfer rates measured here for APC.

142 The full distribution of primarily energy transfer timescales spans $\sim 65$ fs to $\sim 800 \mathrm{fs}$. In ensemble 143 measurements with high temporal resolution, sub-50 fs energy transfer was also observed [19, 21 , 144 22]. This population is absent here, likely due to the longer pulse durations used (100-200 fs) (SI 145 Section 5.5). The few measured timescales above $\sim 400$ fs arise from the effect of Poissonian noise, 146 which elongate the tail of SM2P distributions [38]. The lack of a significant population slower than $147 \sim 400$ fs suggests that static conformational states of the protein do not often dramatically slow the 148 energy transfer timescales. The effect of heterogeneity in the local protein dielectric environment on 149 the distribution of energy transfer timescales was previously investigated theoretically. Using combined 150 quantum chemical/molecular mechanical simulations, a 4-fold range of energy transfer times was found 
151 for another photosynthetic light-harvesting protein, which is roughly comparable with the measured range

152 shown in Fig. 2a [39].

\section{Energetic relaxation in APC}

154 Upon photoexcitation, the chromophores in APC undergo energetic relaxation (red-shifting) due to 155 nuclear motion including intramolecular vibrational relaxation (IVR) and solvation within the protein 156 pocket [40]. The ultrafast solvation, known as inertial solvation, involves coupling to the short-range 157 motions of nearby amino acid side chains and solvent molecules. The collective reorganization of solvent

158 molecules (diffusive solvation) occurs on longer timescales than the picosecond window investigated here 159 [41]. The combined effects of IVR and solvation give rise to the Stokes shift, which moves population 160 out of resonance with the laser pulse. This energetic relaxation can be investigated using the histograms 161 where no energy transfer is present. For the $\alpha$-excitation data, the histograms of timescales from bright 162 (Fig. 2c) and quenched (Fig. 2d) APC have similar median values (257 fs and $276 \mathrm{fs}$, respectively), 163 which is consistent with previous pump-probe experiments that measured a $250 \mathrm{fs}$ Stokes shift [23].

164 The spectral dependence of the energetic relaxation can be examined by comparing the $\alpha$-excitation data 165 to the $\beta$-excitation data for quenched APC. Similar median timescales of $308 \mathrm{fs}$ for the $\beta$-excitation 166 data and $\sim 260$ fs for the $\alpha$-excitation data were found. Consistently, previous measurements found 167 comparable timescales of energetic relaxation for both chromophores, although two components at 120

168 fs and $230 \mathrm{fs}$ were observed [23]. While the median timescales are consistent with the longer of the two 169 components, the shorter one is absent. This may be due to the coherent excitation scheme used in the 170 ensemble measurements or the second component may be hidden in the width of the distribution [42].

\section{Environment-dependent heterogeneity in energetic relaxation}

172 The influence of the protein environment on the distribution of energetic relaxation timescales was in173 vestigated by comparing the distributions for APC, CPC, and Atto647N. CPC is another cyanobacterial 174 light-harvesting protein that is homologous to an APC monomer with an additional peripheral chro175 mophore (SI Section 3, Fig. S4) [31, 36]. There are large distances between the chromophores in CPC, 176 and so energy transfer is slower than the time window measured here [20,43]. Therefore, only energetic 


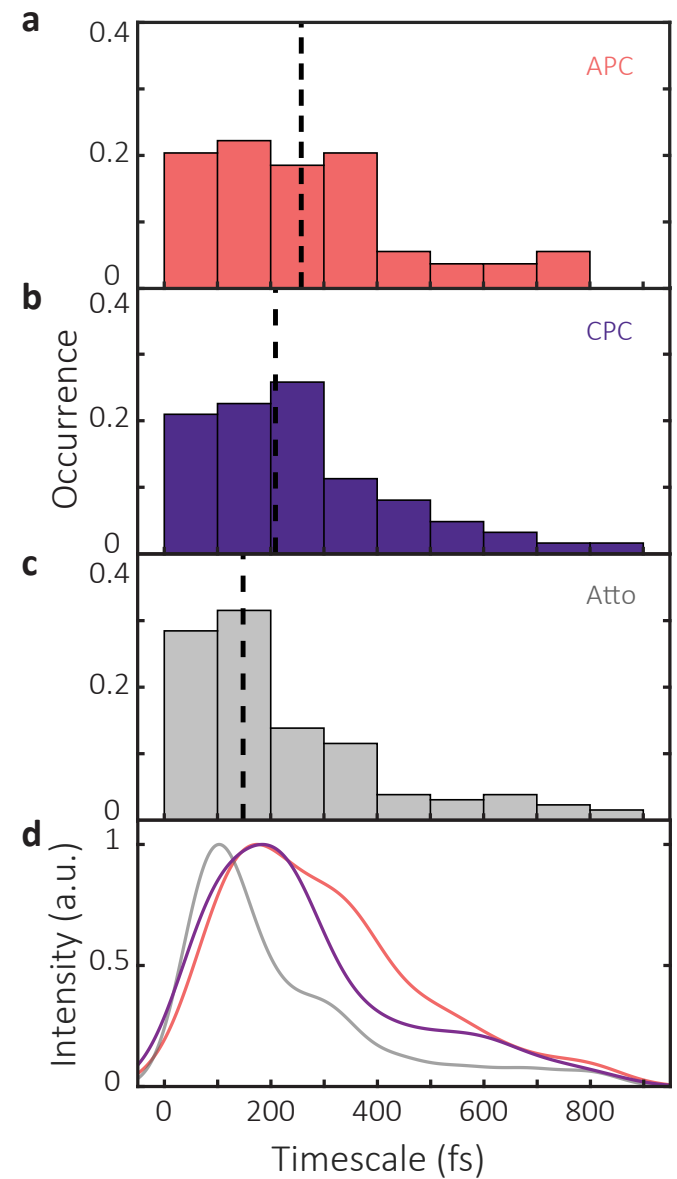

Figure 3: Comparison of histograms of energy relaxation timescales. Histograms of energy relaxation timescales for (a) APC with a $645 \mathrm{~nm}$ excitation, (b) CPC, and (c) Atto647N. The median values of each data set are marked with a dashed line at (a) $258 \mathrm{fs}$, (b) $216 \mathrm{fs}$, and (c) $148 \mathrm{fs}$. The kernel density estimation (KDE) smoothed fit for each histogram is shown in $(\mathbf{d})$.

177 relaxation is present in the distribution. Atto647N is a widely-used single-molecule chromophore with

178 sufficient photostability for SM2P, which the chromophores in APC and CPC lack. Histograms of the 179 measured energetic relaxation timescales are shown in Fig. 3 for APC with $\alpha$ excitation (a), CPC (b)

180 and Atto647N (c). Statistical parameters for all three distributions are given in SI Table S2, which show

181 differences in both the peak locations and widths.

182 A comparison of the distribution for Atto647N to the distributions for APC and CPC isolates the 183 contribution of a protein scaffold, as Atto647N was embedded in a polymer matrix with a local aqueous 184 environment. The asymmetric distribution and median timescale (148 fs) for energetic relaxation of 185 Atto647N was similar to previous measurements of other chromophores in solution. Based on the 186 previous work, we assign the measured energetic relaxation in Atto647N to IVR with a contribution on 
187 the short timescales from electronic dephasing $[13,14]$. The median timescales for energetic relaxation 188 was $258 \mathrm{fs}$ for APC and 216 fs for CPC. The chromophores within APC and CPC are both surrounded by 189 a protein environment, and so the slower timescales than for Atto647N suggest that the protein matrix 190 stabilizes the excited chromophore and its immediate environment to slow relaxation processes such as 191 IVR.

192 The median timescales for APC and for CPC are similar, consistent with their homologous protein struc193 tures. The median value for APC (258 fs) is, however, longer than for CPC (216 fs). Correspondingly, 194 the chromophores in trimeric APC (Fig. 1b) are more buried within the protein than those in monomeric 195 CPC (Fig. S4). The solvent accessible surface area (SASA) for chromophores in CPC $\left(300 \AA^{2}\right)$ is twice 196 that for chromophores in APC $\left(150 \AA^{2}\right)$. The increased solvent accessibility in CPC may create a more 197 solution-like environment that speeds up the energetic relaxation, as observed for Atto647N. Ensemble 198 ultrafast measurements compared the relaxation timescales of chromophore-containing photosynthetic 199 proteins from cryptophytes with open and closed protein scaffolds, which have correspondingly more and 200 less solvent accessibility. These measurements showed faster relaxation for the open scaffolds, consistent 201 with the results described here [44]. Other studies found that the dynamics of CPC were independent 202 of bulk solvent, initially in contradiction with a dependence of relaxation on solvent accessibility [40].

203 However, the local chromophore environment, i.e., the first solvation shell, may not fully reflect changes 204 to the bulk due to interactions such as hydrogen bonding between the protein and water molecules [45].

205 In addition to differences in the median, the distributions of timescales can be used to compare the 206 heterogeneity in energetic relaxation. In SM2P, simulations have shown that the distributions are 207 extended towards longer timescales (here, values $>500 \mathrm{fs}$ ) due to sampling of Poissonian-distributed 208 data [38]. To compare the heterogeneity shown in the distributions, each one was smoothed using 209 a kernel density estimation (Fig. 3d) and the full-width half maximum (FWHM) of the smoothed 210 curves was calculated, which describes the width of the main peak (SI Fig. S9). The distribution for 211 Atto647N (FWHM=179 fs) is much narrower than the distributions for $A P C$ (FWHM=462 fs) and $212 \mathrm{CPC}(\mathrm{FWHM}=295 \mathrm{fs})$. The width for Atto647N is similar to that measured previously for a series of 213 other chromophores in solution, where the width was ascribed to a bimodal profile from the electronic 214 dephasing and IVR contributions $[13,14,38]$. The wider distributions for APC and CPC suggest that, 215 in addition to slowing energetic relaxation, the protein matrix introduces more heterogeneity in the 
relaxation timescale than is present in an aqueous solution.

217 The distribution of energetic relaxation timescales is also broader for APC (FWHM=462 fs) than for CPC

$218(\mathrm{FWHM}=295 \mathrm{fs}$ ). The breadth for APC may arise from increased interaction with the protein due to the

219 more buried position of its chromophores. The distribution for APC has signatures of a bimodal structure

220 with peaks at $\sim 175$ fs and $\sim 375$ fs, along with an elongated tail due to Poissonian noise as discussed

221 above. In contrast, the distribution for CPC has only a single broad peak with a tail. The two peaks in

222 the distribution for APC may correspond to the previously hypothesized two conformations responsible

223 for the $220 \mathrm{fs}$ and $280 \mathrm{fs}$ timescales of energy transfer from ensemble measurements [22]. Alternately,

224 previous measurements found a 400 fs component of energetic relaxation exclusively associated with

225 the $\alpha$ chromophore in APC [23]. Although this component only appeared clearly upon excitation of the

226 vibronic transition in ensemble measurements, which we do not excite here, signatures of this pathway

227 may be contributing to the width of the measured distribution.

\section{Discussion}

229 In this work, we described spectrally tunable SM2P and the use of this tunability to disentangle the 230 ultrafast dynamics of APC at the single-protein level. The distribution of primarily energy transfer

231 timescales for APC is clustered around the median at $\sim 150 \mathrm{fs}$, whereas the mean - and potentially 232 ensemble values - were lengthened due to the influence of small subpopulations. Furthermore, previous 233 assignments of complex dynamics could instead arise from the microscopic heterogeneity, i.e., non234 Gaussian distribution, observed here.

235 Slow and heterogeneous energetic relaxation timescales for APC and CPC indicated that the protein 236 matrix introduces variation in the photophysical processes. Because energetic relaxation is slower than 237 energy transfer, the states involved in energy transfer are the initially excited ones, which may be how 238 rapid energy transfer is maintained despite the effects of heterogeneous energetic relaxation. It may

239 also be that slow energetic relaxation in the protein helps maintain vibronic coupling through an energy

240 transfer event, which is thought to mediate rapid transfer [46]. The ability of the protein to influence

241 the timescales of energetic relaxation means that the protein structure can control the excited states 242 involved in energy transfer, and even the mechanism of energy transfer itself. 


\section{Methods}

244 Single-molecule pump-probe (SM2P) spectroscopy. A schematic of the experimental setup is shown

245 in the SI Fig. S1 and is described in detail in Ref. [38]. In brief, a tunable fiber laser (FemtoFiber pro,

246 Toptica; 120 fs pulse duration, $\sim 4 \mathrm{~nm}$ bandwidth, $80 \mathrm{MHz}$ repetition rate) was used as the excitation

247 source and the laser repetition rate was reduced from $80 \mathrm{MHz}$ to $2.5 \mathrm{MHz}$ or $312.5 \mathrm{KHz}$ with an acousto-

248 optic pulse picker (Brimrose Corporation, FSPP-400-80-BR-800). The prism compressor was designed

249 with single-axis translation for easy optimization with spectral maximum. The compressor was translated

250 to minimize dispersion for each center wavelength as measured at the sample position with an inter-

251 ferometric autocorrelation using a GaP photodiode (Marktech, MTPD3650D-1.4) [47]. Representative

252 intensity autocorrelations for different laser excitations are shown in SI Fig. S2 with the FHWM deter-

253 mined assuming a Gaussian pulse. The laser pulses were split by a set of 50/50 beam splitters (Thorlabs,

254 UFBS5050) and a delay stage in a Mach-Zehnder interferometer. The polarization was converted from

255 horizontal to cicular polarization using a quarter-wavplate (Newlight Photonics, WPM03-Q-VIS). The

256 excitation was coupled into a custom-designed inverted confocal microscope (Mad City labs, RM21)

257 and focused onto the sample with an objective (UPLSAPO100XO, Olympus, NA 1.4). Fluorescence

258 emission was collected through the same objective and separated from the excitation by use of dichroic

259 mirrors optimized for excitation filtering (Chroma, T635Ipxr-UF3; Chroma, ZT647rdc-UF2) and a pair

260 of bandpass filters (Semrock, FF02-675/67-25; Chroma, ET690/120x) Fluorescence photons were de-

261 tected on an avalanche photodiode (SPCM-AQRH-15, Excelitas) with a time-correlated single photon

262 counting module (TimeTagger20, Swabian Instruments). Fluorescence lifetimes were fit to a single

263 exponential decay convolved with an experimentally determined instrument response function $(\sim 0.5$

264 ns) as described in SI Section 3.

265 For SM2P experiments, the power was set to $\sim 1 \mathrm{pJ} /$ pulse before the objective or $700 \frac{\mu \mathrm{J}}{\mathrm{cm}^{2}}$ per pulse 266 at the sample plane. The center wavelength of the laser was tuned to either $610 \mathrm{~nm}$ or $645 \mathrm{~nm}$.

267 For the $610 \mathrm{~nm}$ measurements, experiments were performed with both near-Fourier transform limited 268 temporal compression (118 fs) and no temporal compression (180 fs). The measured dynamics were 269 independent of pulse duration (Fig. S7). For the $645 \mathrm{~nm}$ measurements, experiments were performed 270 with no temporal compression (300 fs) due to power restraints. The delay time between the two pulses 271 was scanned from $-1.5 \mathrm{ps}$ to $1.5 \mathrm{ps}$ at $100 \mu \mathrm{m} / \mathrm{s}(0.33 \mathrm{ps} / \mathrm{s})$. Fluorescence emission was binned into 
$27250 \mathrm{~ms}$ bins before being fit with maximum likelihood estimation to extract energy relaxation timescales.

273 The fit function was the convolution of the measured intensity autocorrelation with an exponential rise

274 function for energy relaxation as detailed in SI Section $1[13,14,16]$. The standard error was estimated 275 using the Fisher information matrix [48]. Single-molecule blinking and on-off transitions in SM2P traces

276 were identified by eye and omitted from the data analysis.

277 Energy relaxation timescales were used to construct histograms with the bin width determined by the

278 Freedman-Diaconis rule. The generated distributions were compared using a Permutation test, which 279 determines the likelihood of randomly allocating the complete data-set into two groups and obtaining 280 the experimentally observed difference (SI Section 5.2). SASA values were determined using the 'Protein 281 interfaces, surfaces and assemblies' service PISA at the European Bioinformatics Institute located online 282 at (http://www.ebi.ac.uk/pdbe/prot_int/pistart.html) [49].

283 Sample preparation. APC (Sigma Aldrich, S868), CPC, (Agilent, PB11), and Atto647N (Ther284 moFisher, 04507-1MG-F) were purchased and diluted to $\sim 1 \mathrm{nM}$ in $\mathrm{pH} 7.4$ phosphate buffer solution 285 (ThermoFisher, AM9624). An enzymatic oxygen-scavenging system was added to the solution at final 286 concentrations of $25 \mathrm{nM}$ protocatechuate-3,4-dioxygenase and $2.5 \mathrm{mM}$ protocatechuic acid [50]. The 287 solution was spincoated in 1\% PVA onto glass coverslips, which were placed on a piezoelectric stage 288 (Mad City labs, Nano-LP100) on the microscope.

\section{Data Availability}

290 The data that support the findings of this study are available from the corresponding author upon 291 request.

\section{Acknowledgments}

293 This work was supported by the NIH Director's New Innovator Award 1DP2GM128200-01 and a Beck-

294 man Young investigator Award (to G.S.S.-C.). R.M. acknowledges an NSF Graduate Research Fellow295 ship. G.S.S.-C. also acknowledges a Smith Family Award for Excellence in Biomedical Research, Sloan 296 Research Fellowship in Chemistry and a CIFAR Global Scholar Award. 


\section{Author Contributions}

298 R.M., T.K., and G.S.S.-C. conceived and designed the experiments. R.M. and A.N. performed the 299 experiments. R.M. and G.S.S.-C. analysed the data. R.M., A.N., and G.S.S.-C. co-wrote the paper. All

300 authors discussed the results and commented on the manuscript.

\section{Competing financial interests}

302 The authors declare no competing financial interests.

\section{References}

304 1. Blankenship, R. E. Molecular Mechanisms of Photosynthesis (John Wiley \& Sons, 2014).

305 2. Ishizaki, A. \& Fleming, G. R. Unified treatment of quantum coherent and incoherent hopping 306 dynamics in electronic energy transfer: Reduced hierarchy equation approach. J. Chem. Phys. 130, 234111 (2009).

308

3. Ishizaki, A., Calhoun, T. R., Schlau-Cohen, G. S. \& Fleming, G. R. Quantum coherence and its interplay with protein environments in photosynthetic electronic energy transfer. Phys. Chem. Chem. Phys. 12, 7319-7337 (2010).

4. Novoderezhkin, V. I. \& van Grondelle, R. Physical origins and models of energy transfer in photosynthetic light-harvesting. Phys. Chem. Chem. Phys. 12, 7352-7365 (2010).

5. Şener, M., Strümpfer, J., Hsin, J., Chandler, D., Scheuring, S., Hunter, C. N. \& Schulten, K. Förster energy transfer theory as reflected in the structures of photosynthetic light-harvesting systems. Chem. Phys. Chem 12, 518-531 (2011).

6. Scholes, G. D. Long-range resonance energy transfer in molecular systems. Annu. Rev. of Phys. Chem. 54, 57-87 (2003).

7. Van Amerongen, H., Valkunas, L. \& van Grondelle, R. Photosynthetic Excitons (World Scientific, Singapore, 2000). 
8. Blankenship, R. E., Tiede, D. M., Barber, J., Brudvig, G. W., Fleming, G. R., Ghirardi, M., Gunner, M. R., Junge, W., Kramer, D. M., Melis, A., Moore, T. A., Nozik, A. J., Ort, D. R., Parson, W. W., Prince R. C.and Moser, C. C., Nocera, D. G. \& Sayre, R. T. Comparing photosynthetic and photovoltaic efficiencies and recognizing the potential for improvement. Science 332, 805-809 (2011).

9. Lerner, E., Cordes, T., Ingargiola, A., Alhadid, Y., Chung, S., Michalet, X. \& Weiss, S. Toward dynamic structural biology: Two decades of single-molecule Förster resonance energy transfer. Science 359 (2018).

10. Kondo, T., Chen, W. J. \& Schlau-Cohen, G. S. Single-molecule fluorescence spectroscopy of photosynthetic systems. Chem. Rev. 117, 860-898 (2017).

11. Beane, G., Devkota, T., Brown, B. S. \& Hartland, G. V. Ultrafast measurements of the dynamics of single nanostructures: a review. Rep. Prog. Phys. 82, 016401 (2018).

12. Cogdell, R. J., Gall, A. \& Köhler, J. The architecture and function of the light-harvesting apparatus of purple bacteria: from single molecules to in vivo membranes. Quart. Rev. Biophys. 39, 227-324 (2006).

13. Van Dijk, E. M., Hernando, J., Garcıa-López, J.-J., Crego-Calama, M., Reinhoudt, D. N., Kuipers, L., Garcıa-Parajó, M. F. \& van Hulst, N. F. Single-molecule pump-probe detection resolves ultrafast pathways in individual and coupled quantum systems. Phys. Rev. Lett. 94, 078302 (2005).

14. Van Dijk, E., Hernando, J., Garcia-Parajó, M. \& Van Hulst, N. Single-molecule pump-probe experiments reveal variations in ultrafast energy redistribution. J. Chem. Phys. 123, 064703 (2005).

15. Hernando, J., van Dijk, E. M. H. P., Hoogenboom, J. P., García-López, J., Reinhoudt, D., CregoCalama, M., García-Parajó \& van Hulst, N. F. Effect of disorder on ultrafast exciton dynamics probed by single molecule spectroscopy. Phys Rev. Lett. 97, 216403 (2006).

16. Malý, P., Gruber, J. M., Cogdell, R. J., Mančal, T. \& van Grondelle, R. Ultrafast energy relaxation in single light-harvesting complexes. Proc. Natl. Acad. Sci. 113, 2934-2939 (2016).

17. Hildner, R., Brinks, D., Nieder, J. B., Cogdell, R. J. \& van Hulst, N. F. Quantum Coherent Energy Transfer over Varying Pathways in Single Light-Harvesting Complexes. Science 340, 1448-1451 (2013). 
348 18. Brinks, D., Stefani, F. D., Kulzer, F., Hildner, R., Taminiau, T. H., Avlasevich, Y., Müllen, K. \& Van Hulst, N. F. Visualizing and controlling vibrational wave packets of single molecules. Nature

351 19. Womick, J. M. \& Moran, A. M. Vibronic enhancement of exciton sizes and energy transport in photosynthetic complexes. J. Phys. Chem. B 115, 1347-1356 (2011).

353 20. Womick, J. M. \& Moran, A. M. Nature of excited states and relaxation mechanisms in Cphycocyanin. J. Phys. Chem. B 113, 15771-15782 (2009).

355 21. Womick, J. M., Miller, S. A. \& Moran, A. M. Toward the origin of exciton electronic structure in phycobiliproteins. J. Chem. Phys. 133, 07B603 (2010).

357 22. Womick, J. M. \& Moran, A. M. Exciton coherence and energy transport in the light-harvesting 358 dimers of allophycocyanin. J. Phys. Chem. B 113, 15747-15759 (2009).

359 23. Edington, M. D., Riter, R. E. \& Beck, W. F. Femtosecond transient hole-burning detection of 360 interexciton-state radiationless decay in allophycocyanin trimers. J. Phys. Chem. B 101, 44734477 (1997).

362

24. Edington, M. D., Riter, R. \& Beck, W. F. Interexciton-state relaxation and exciton localization in allophycocyanin trimers. J. Phys. Chem. 100, 14206-14217 (1996).

25. Edington, M. D., Riter, R. E. \& Beck, W. F. Evidence for coherent energy transfer in allophycocyanin trimers. J. Phys. Chem. 99, 15699-15704 (1995).

366 26. Beck, W. F. \& Sauer, K. Energy-transfer and exciton-state relaxation processes in allophycocyanin. J. Phys. Chem. 96, 4658-4666 (1992).

368 27. Riter, R. R., Edington, M. D. \& Beck, W. F. Protein-matrix solvation dynamics in the $\alpha$ subunit of C-phycocyanin. J. Phys. Chem. 100, 14198-14205 (1996).

370 28. Homoelle, B. J., Edington, M. D., Diffey, W. M. \& Beck, W. F. Stimulated photon-echo and 371 transient-grating studies of protein-matrix solvation dynamics and interexciton-state radiationless decay in $\alpha$ phycocyanin and allophycocyanin. J. Phys. Chem. B 102, 3044-3052 (1998).

373 29. Goldsmith, R. \& Moerner, W. Watching conformational- and photodynamics of single fluorescent proteins in solution. Nat. Chem. 2, 179-185 (2010). 
375 30. Wang, Q. \& Moerner, W. Dissecting pigment architecture of individual photosynthetic antenna complexes in solution. Proc. Natl. Acad. Sci. 112, 13880-13885 (2015).

377 31. Squires, A. H. \& Moerner, W. Direct single-molecule measurements of phycocyanobilin photophysics in monomeric C-phycocyanin. Proc. Natl. Acad. Sci. 114, 9779-9784 (2017).

379

380

381

382

383 384

32. Gwizdala, M., Berera, R., Kirilovsky, D., Van Grondelle, R. \& Krüger, T. P. Controlling light harvesting with light. J. Am. Chem. Soc. 138, 11616-11622 (2016).

33. Ying, L. \& Xie, X. S. Fluorescence spectroscopy, exciton dynamics, and photochemistry of single allophycocyanin trimers. J. Phys. Chem. B 102, 10399-10409 (1998).

34. Loos, D., Cotlet, M., De Schryver, F., Habuchi, S. \& Hofkens, J. Single-molecule spectroscopy selectively probes donor and acceptor chromophores in the phycobiliprotein allophycocyanin. Biophys. J. 87, 2598-2608 (2004).

35. Brejc, K., Ficner, R., Huber, R. \& Steinbacher, S. Isolation, crystallization, crystal structure analysis and refinement of allophycocyanin from the cyanobacterium Spirulina platensis at 2.3 Åresolution. J. Molec. Biol. 249, 424-440 (1995).

36. MacColl, R. Cyanobacterial phycobilisomes. J. Struct. Biol 124, 311-334 (1998).

37. Mohseni, M., Rebentrost, P., Lloyd, S. \& Aspuru-Guzik, A. Environment-assisted quantum walks in photosynthetic energy transfer. J. Chem. Phys. 129, 11B603 (2008).

38. Moya, R., Kondo, T., Norris, A. \& Schlau-Cohen, G. S. Spectrally-tunable femtosecond singlemolecule pump-probe spectroscopy. In Preparation.

39. Curutchet, C., Kongsted, J., Munoz-Losa, A., Hossein-Nejad, H., Scholes, G. D. \& Mennucci, B. Photosynthetic light-harvesting is tuned by the heterogeneous polarizable environment of the protein. J. Am. Chem. Soc. 133, 3078-3084 (2011).

40. Homoelle, B. J. \& Beck, W. F. Solvent Accessibility of the Phycocyanobilin chromophore in the $\alpha$ subunit of C-phycocyanin: implications for a molecular mechanism for inertial protein-matrix solvation dynamics. Biochemistry 36, 12970-12975 (1997).

41. Stratt, R. M. \& Cho, M. The short-time dynamics of solvation. J. Chem. Phys. 100, 6700-6708 (1994). 
402 42. Ferwerda, H. A., Terpstra, J. \& Wiersma, D. A. Discussion of a "coherent artifact" in four-wave 403 mixing experiments. J. Chem. Phys. 91, 3296-3305 (1989).

404 43. Riter, R. E., Edington, M. D. \& Beck, W. F. Isolated-chromophore and exciton-state photophysics 405 in C-phycocyanin trimers. J. Phys. Chem. B 101, 2366-2371 (1997).

406 44. Jumper, C. C., Arpin, P. C., Turner, D. B., McClure, S. D., Rafiq, S., Dean, J. C., Cina, J. A., 407 Kovac, P. A., Mirkovic, T. \& Scholes, G. D. Broad-band pump-probe spectroscopy quantifies 408 ultrafast solvation dynamics of proteins and molecules. J. Phys. Chem. Lett. 7, 4722-4731 (2016).

409 45. Biedermannová, L. \& Schneider, B. Hydration of proteins and nucleic acids: Advances in experiment 410 and theory. A review. Biochim. Biophys. Acta Gen. Subj. 1860, 1821-1835 (2016).

411 46. Jumper, C. C., Rafiq, S., Wang, S. \& Scholes, G. D. From coherent to vibronic light harvesting in 412 photosynthesis. Curr. Opin. Chem. Biol. 47, 39-46 (2018).

413 47. Müller, M., Squier, J. \& Brakenhoff, G. Measurement of femtosecond pulses in the focal point of 414 a high-numerical-aperture lens by two-photon absorption. Opt. Lett. 20, 1038-1040 (1995).

415 48. Pawitan, Y. In all likelihood: statistical modelling and inference using likelihood (Oxford University 416 Press, 2001).

417 49. Krissinel, E. \& Henrick, K. Inference of macromolecular assemblies from crystalline state. J. Mol. $418 \quad$ Bio. 372, 774-797 (2007).

419 50. Aitken, C. E., Marshall, R. A. \& Puglisi, J. D. An oxygen scavenging system for improvement of 420 dye stability in single-molecule fluorescence experiments. Biophys. J. 94, 1826-1835 (2008). 
Figures

a

$$
|\Delta T|<\tau_{E R} \text { (dimmer) }
$$

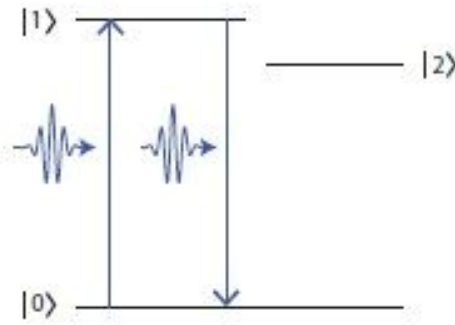

b

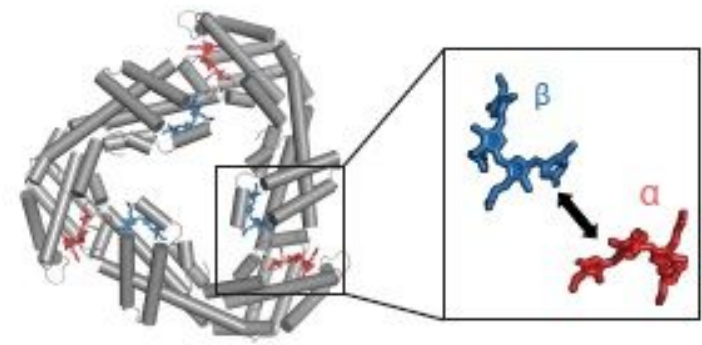

d

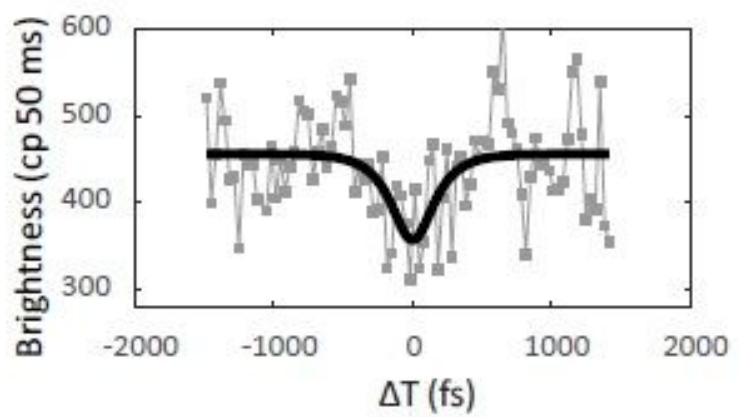

f

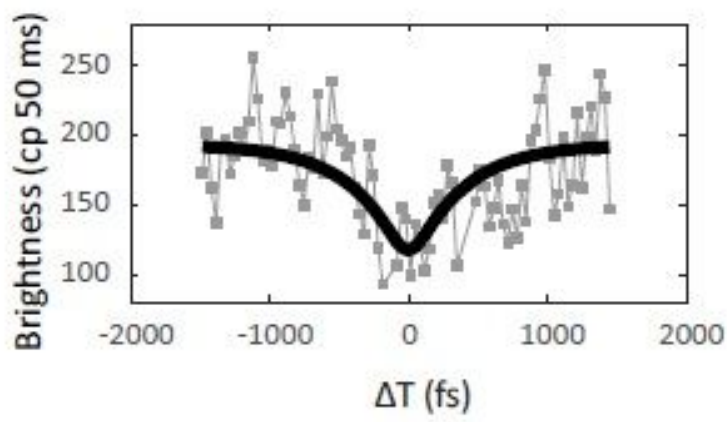

$|\Delta T|>\tau_{E R}$ (brighter)

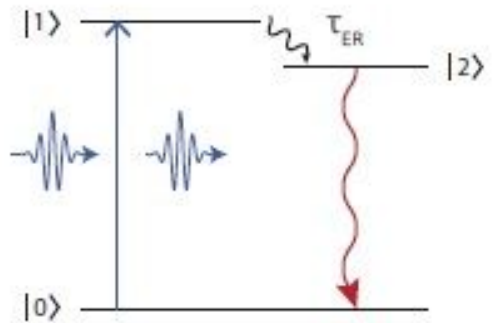

c

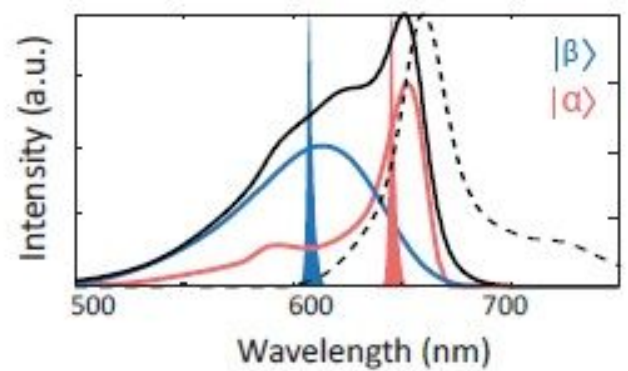

e

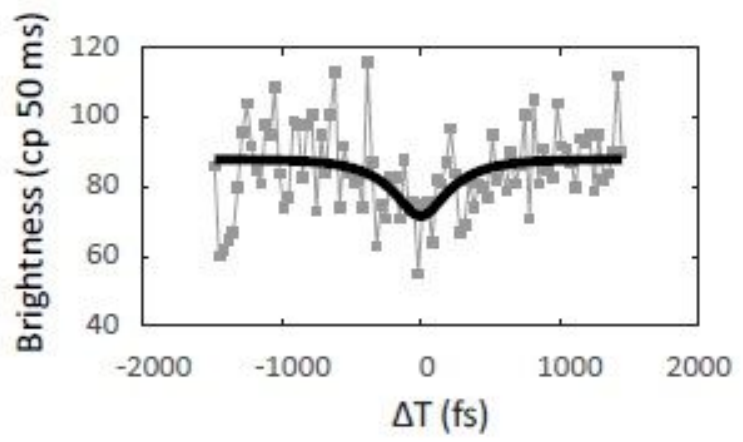

g

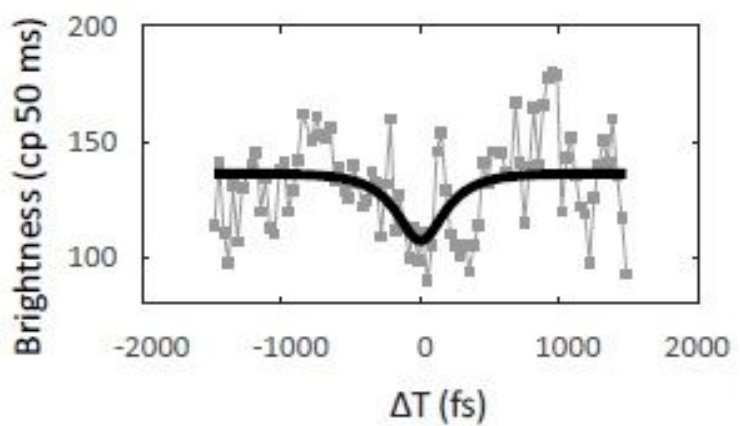

Figure 1

Single-molecule pump probe (SM2P) experiments on allophycocyanin (see Manuscript file for complete figure legend) 
c
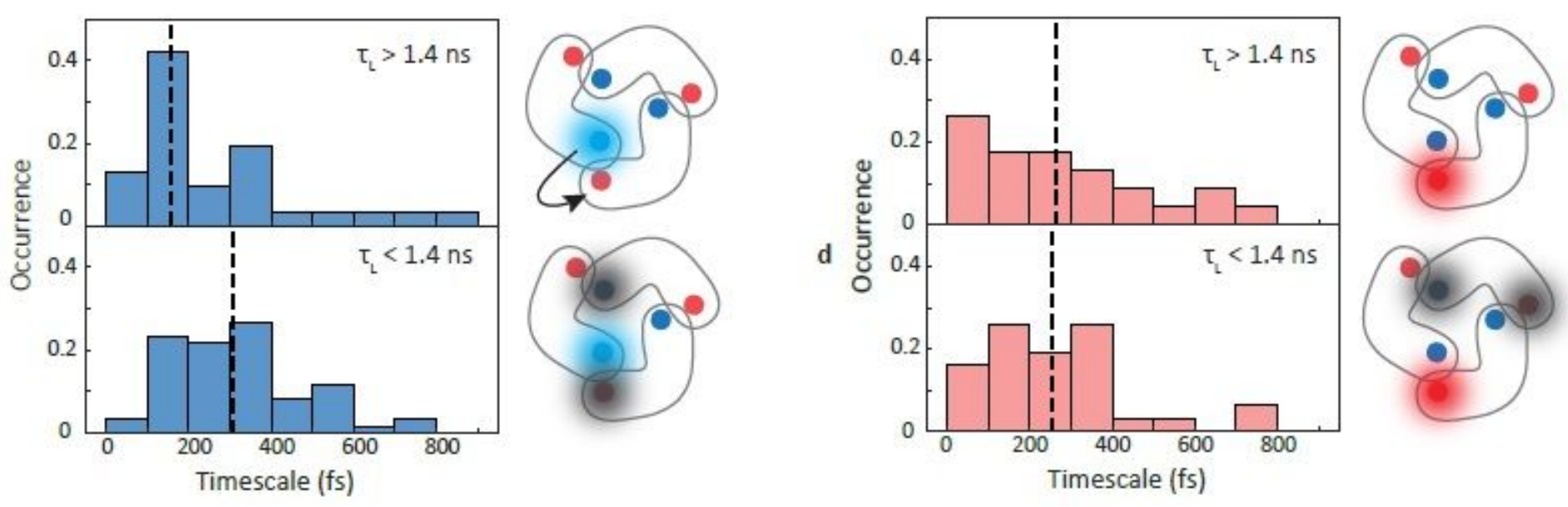

Figure 2

Distributions of energetic relaxation and energy transfer timescales for APC. (see Manuscript file for complete figure legend) 


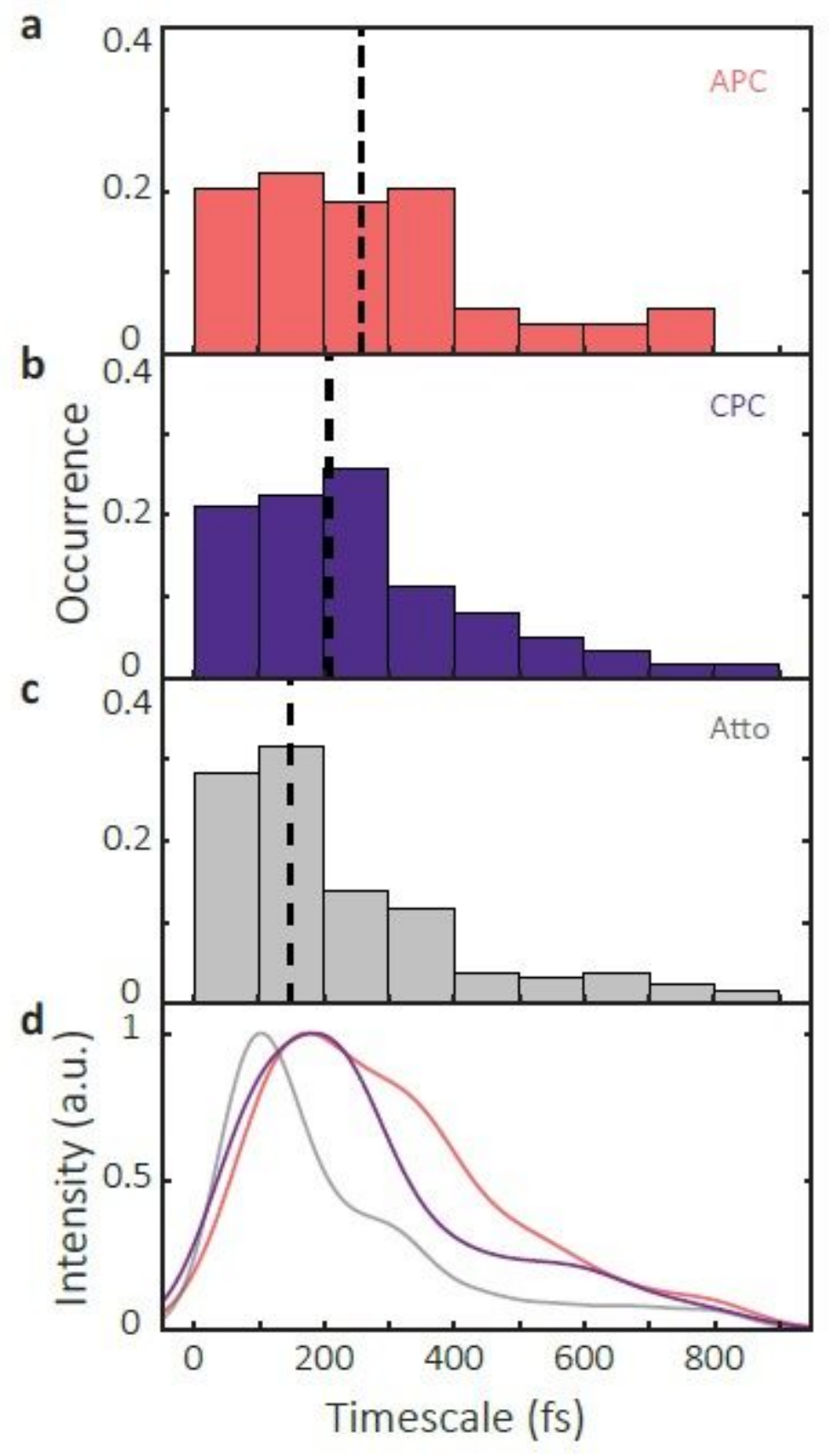

Figure 3

Comparison of histograms of energy relaxation timescales. (see Manuscript file for complete figure legend)

\section{Supplementary Files}

This is a list of supplementary files associated with this preprint. Click to download.

- MoyaAPCSIV02.pdf 